Société d'histoire de la révolution de 1848 et des

révolutions du XIXe siècle

$47 \mid 2013$

Quel est l'avenir du XIX ${ }^{\mathrm{e}}$ siècle ?

\title{
Artiste, la société a besoin de toi !
}

\section{Georges Buisson}

URL : http://journals.openedition.org/rh19/4549

DOI : 10.4000/rh19.4549

ISSN : $1777-5329$

Éditeur

La Société de 1848

Édition imprimée

Date de publication : 31 décembre 2013

Pagination : 59-65

ISSN : 1265-1354

Référence électronique

Georges Buisson, «Artiste, la société a besoin de toi ! », Revue d'histoire du XIXe siècle [En ligne],

47 | 2013, mis en ligne le 31 décembre 2016, consulté le 20 avril 2019. URL : http://

journals.openedition.org/rh19/4549; DOI : 10.4000/rh19.4549 


\section{GEORGES BUISSON}

\section{Artiste, la société a besoin de toi!}

L'avenir du XIX siècle, s'il devait en avoir un, serait de remettre dans la baignoire l'eau du bain avec le bébé, qu'un peu trop vite, le siècle suivant a jeté avec. Le XIXe, siècle des colères et des combats, donc siècle d'avenir, a réussi à inscrire dans la réalité actuelle de notre pays tant d'améliorations, de clarifications et de progrès que l'on a pu se dire : «ça y est, cette fois nous avons avancé et nous ne reviendrons plus en arrière!» Et pourtant...

Il aura fallu, par exemple, une centaine d'années pour que notre société s'affranchisse du poids de la religion dominante qu'a été, à cette époque, le catholicisme. Ce combat rude et courageux aboutit à la juste séparation de l'Église et de l'État, à l'instauration d'une laïcité garantissant la séparation définitive des pouvoirs. Mais certains ne reparlent-ils pas aujourd'hui de la nécessité de revenir à une laïcité plus "ouverte» ou plus "généreuse »? N'estce pas là une tentative qui souhaiterait remettre les règles religieuses au-dessus des lois de la République?

Le XIX ${ }^{\mathrm{e}}$ siècle fut le siècle des utopies et des ruptures. Il nous rappelle la nécessité de résister à tout renoncement. Ces combats d'émancipation pour le progrès et la liberté malgré tous les soubresauts implacables qu'ils ont produits, ont été en parti nourris de la pensée des intellectuels en général et des écrivains en particulier. Sand, Hugo, Dickens, Zola, pour n'en citer que quelques uns, ont contribué par leurs écrits à animer les esprits et à œuvrer volontairement à la progression de la pensée.

Pendant plus de dix ans, j'ai eu la belle responsabilité d'administrer, pour le Centre des Monuments nationaux, la demeure de George Sand. La romancière avait fait, bien avant l'heure, de sa maison, un centre culturel de rencontre. Nohant réunit artistes, philosophes, hommes politiques dans un creuset fécond, libre et inventif. Pourtant le XXe siècle fit de George Sand la «bonne dame de Nohant", celle que la bourgeoisie rendit fréquentable en gommant l'écrivain engagé politiquement qu'elle fut et en mettant en avant l'image d'Épinal de la grand-mère confiture au détriment de la femme libre et indépendante qu'elle était aussi. Le camp progressiste ne fut pas plus tendre à son égard, lui reprochant, plus qu'à n'importe quel autre écrivain, son incompréhension et son 
rejet de la Commune de Paris. Enfin le mouvement féministe, sous la plume de Simone de Beauvoir, qualifia de «mensonge» son engagement féministe. Le $\mathrm{XX}^{\mathrm{e}}$ siècle a donc été particulièrement injuste avec celle qui avait mis sa plume au service de ses idées. Il préféra retenir la posture de l'artiste sacralisé, souvent marginalisé, celui dont l'œuvre serait vierge de toute compromission avec son environnement social et politique. Il enterra avec Jean-Paul Sartre les derniers grands artistes engagés, les héritiers de ce XIX ${ }^{\mathrm{e}}$ siècle qui paraissait bel et bien dépassé. Pourtant tous ces écrivains, bien au-delà d'une simple conception de l'art pour l'art, avaient engagé le combat des idées en revendiquant une œuvre émancipatrice. George Sand ne disait pas autre chose en affirmant avec lucidité dans une lettre à Emile Aucante le 16 mars 1851 :

«Tout ce qui était en moi, je l'ai dit, je l'ai mis, à mes risques et périls, dans une longue série de romans que les éditeurs n’ont jamais su ni voulu populariser. J'ai blessé la bourgeoisie qui me lisait, je n'ai pas instruit le peuple qui ne pouvait pas me lire. Pour utiliser enfin le peu d'utilité que j'ai pu mettre dans mes écrits, je me décide à publier à mes frais mes ouvres complètes à 4 sous la livraison. J'y gagnerai ou j'y perdrai ma petite fortune littéraire, peu importe. J'aurai fait mon possible pour mettre à la portée de tous ceux qui savent et veulent lire, des écrits que je n'estime pas plus qu'ils ne valent, mais où j'ai fait tout ce qui était possible pour instruire et moraliser les diverses classes de la Société.»

Le débat intellectuel qui anima la correspondance qu'elle échangea avec Flaubert ne dit pas autre chose. Sand se situe résolument au cœur de la société, malgré son incompréhension de la Commune. Elle le revendique en écrivant à Flaubert le 25 octobre 1872 :

"Je ne plains pas l'incendiaire et l'assassin qui tombent sous le coup de la loi; je plains profondément la classe qu'une vie brutale, déchue, sans essor et sans aide, réduit à produire de pareils monstres. Je plains l'humanité, je la voudrais bonne, parce que je ne peux m'abstraire d'elle, parce qu'elle est moi, parce que le mal qu'elle se fait me frappe au cœur, parce que sa honte me fait rougir, parce que ses crimes me tordent le ventre..."

Ce à quoi Flaubert répond trois jours plus tard :

"Je ne me crois pas cependant un monstre d'égoïsme. Mon moi s'éparpille tellement dans les livres que je passe des journées entières sans le sentir. J'ai de mauvais moments, il est vrai, mais je me remonte par cette réflexion "personne, au moins, ne m'embête". Après quoi je me retrouve d'aplomb.»

De façon encore plus explicite, il avait déjà confié à George Sand dans une lettre en 1868 : 
"Je ne crois même pas que le romancier doive exprimer son opinion sur les choses de ce monde. Il peut la communiquer, mais je n'aime pas à ce qu'il la dise (cela fait partie de ma poétique à moi). Je me borne donc à exposer les choses telles qu'elles me paraissent, à exprimer ce qui me semble le vrai. Tant pis pour les conséquences..."

Il insiste encore, comme pour exprimer son total dépit, dans une autre lettre le 8 septembre 1871 :

«Le premier remède serait d'en finir avec le suffrage universel, la honte de l'esprit humain..."

Plus loin dans sa correspondance, il poursuit :

«Ah! Chère bon maître, si vous pouviez haïr! C'est là ce qui vous a manqué : la haine. Malgré vos grands yeux de sphinx, vous avez vu le monde à travers une couleur d'or. Elle venait du soleil de votre cœur; mais tant de ténèbres ont surgi, que vous voilà maintenant ne reconnaissant plus les choses. Allons donc! criez! tonnez!...»

Ce à quoi George Sand s'empresse de répondre par retour de courrier :

«Et quoi, tu veux que je cesse d'aimer? Tu veux que je dise que je me suis trompée toute ma vie, que l'humanité est méprisable, haïssable, qu'elle a toujours été, qu'elle sera toujours ainsi? Et tu me reproches ma douleur comme une faiblesse, comme le puéril regret d'une illusion perdue? Tu affirmes que le peuple a toujours été féroce, le prêtre toujours hypocrite, le bourgeois toujours lâche, le soldat toujours brigand, le paysan toujours stupide? Tu dis que tu savais tout cela dès ta jeunesse et tu te réjouis de n'en avoir jamais douté parce que l'âge mûr ne t'a apporté aucune déception; tu n'as donc pas été jeune? Ah! nous différons bien, car je n'ai cessé de l'être si c'est être jeune que d'aimer toujours... [...]

Non, non, on ne s'isole pas, on ne rompt pas les liens du sang, on ne maudit pas, on ne méprise pas son espèce. L'humanité n'est pas un vain mot. Notre vie est faite d'amour et ne plus aimer, c'est ne plus vivre. Le peuple, dis-tu! Le peuple c'est toi et moi, nous nous en défendrions en vain... [...] Le peuple toujours féroce, dis-tu, moi je te dis : La noblesse toujours sauvage!...

Et toi, ami, tu veux que je voie ces choses avec une stoïque indifférence? Tu veux que je te dise : l'homme est ainsi fait; le crime est son expression, l'infamie est sa nature?

Non, cent fois non. L'humanité est indignée en moi et avec moi...»

Et cette très longue lettre, au-delà de la réponse circonstanciée à une relation intime, sera, par les soins de George Sand, rendue publique dans le journal Le Temps le 3 octobre 1871 sous le titre : «Réponse à un ami». 
Ces quelques échanges de vue choisis dans une correspondance s'étalant sur plus de dix années montrent bien combien le milieu des intellectuels en général et des écrivains en particulier participait activement au débat d'idées. On se rappellera «l'investissement par la plume» considérable qui fut celui de George Sand, dans son combat idéologique avant et pendant 1848. Elle n'hésite pas pour cela à devenir journaliste en créant, sur ses fonds propres, plusieurs journaux. Elle participe, en outre, dans l'ombre, à la rédaction des Bulletins de la République qui se voulaient être les passeurs des idées nouvelles de la jeune République vers toutes les classes de la société.

Cette époque pleine de hardiesse n'avait d'ailleurs pas hésité à mettre au sommet de l'État un poète. Ce sera la seule fois de notre histoire. George Sand croit puissamment à l'insertion sociale de l'art et à ses vertus pédagogiques. Elle est aux antipodes de la sacralisation de l'art qui finira par triompher avec ce XIX e siècle finissant. Elle s'est toujours considérée comme une simple "ouvrière de la plume», et n'a jamais craint d'être "instrumentalisée». Elle affirmait : "L'art n'est pas une étude de la réalité positive, c'est une recherche de la vérité idéale...»

George Sand, par conviction, s'opposa donc au naturalisme très majoritaire dans la littérature de son temps, lui préférant ce que l'on pourrait nommer l'idéalisme. Elle n'hésita pas à affronter la statue du commandeur qu'était alors Victor Hugo. Après la lecture des Misérables, elle n'hésite pas à lui écrire le 17 avril 1862 :

"Cette grimace de douleur et d'effroi, je la fais souvent en vous lisant; les désespérances de votre pensée sur la pauvre race humaine me font souvent saigner le cœur. Et $j$ 'ai besoin de me rappeler que vous faites une guerre héroïque et acharnée à nos abominables institutions, répressions à nos impitoyables préjugés, pour m'abstenir de plaintes et même de reproches.»

Victor Hugo se sent ébranlé par cette franchise, il répond :

«Votre lettre m’a attristé. Jugez si ma surprise a été pénible. Je m’étais figuré que ce livre nous rapprocherait, et voici qu'il nous éloigne, qu'il nous désunit presque. J'en voudrais à ce livre si je ne le savais pas si honnête.»

Cette posture de l'artiste engagé, en prise direct avec son temps, qui donne vie dans son œuvre à des gens "comme tout le monde», mais beaux, sert à montrer la société telle qu'elle devrait être, à la rêver, pour sans doute mieux la comprendre. George Sand ne se départit jamais de cette certitude que le monde, en dépit de tout, progressera. À la fin de sa vie, comme une conclusion provisoire sur son parcours, elle écrit : 
"J'ai essayé, dans tout le cours d'une vie laborieuse, non de réformer les idées, au moins d'adoucir les sentiments de mes contemporains. La douloureuse histoire de ce siècle ne prouve pas que j'ai eu sur eux la moindre influence, mais c'est la raison de plus pour continuer, tant que j'aurai un souffle de vie, car n'eussé-je persuadé qu'une seule âme, je n'aurais pas perdu mon temps.»

Pourtant bon nombre d'écrivains, dans ce siècle dont elle parle, étaient farouchement méfiants vis-à-vis du peuple et de la multitude. Ainsi Leconte de Lisle dans une lettre datée du 30 avril 1848 affirmait :

"Que l'humanité est une sale et dégoûtante engeance. Que le peuple est stupide! C'est une éternelle race d'esclaves qui ne peut vivre sans bât et sans joug. "

Et un an plus tard, le 7 septembre 1849 il récidive en exprimant sa méfiance d'artiste :

"Comment l'artiste ne voit-il pas que tous ces hommes voués aux brutalités de l'action, aux divagations banales, aux rabâchages des mesquines et pitoyables théories contemporaines ne sont pas pétris du même limon que le sien?»

Cette méfiance des écrivains vis-à-vis du peuple trouvera son paroxysme après la Commune. Nous ne citerons que Feydeau, qui écrit dans son ouvrage Consolation «L'effronterie de ces coquins n'avait d'égales que leur bêtise et leur scélératesse...", pour démontrer à quel point le rejet de la plupart des artistes pour les masses populaires est viscéral.

Dans la conclusion de son passionnant ouvrage Les écrivains contre la Commune (1970), Paul Lidsky affirme :

"Seuls ont pu échapper à l'empire de la bourgeoisie, soit un grand écrivain comme Hugo dans la mesure où sa popularité lui assurait une certaine indépendance vis-à-vis de son public bourgeois, soit des écrivains bohèmes qui n'avaient pas à redouter ce public bourgeois, puisqu'ils n'avaient donc rien à perdre."

Sans doute pourrions-nous ajouter Sand dans ceux qui conservèrent une posture intellectuelle indépendante.

Son incompréhension pour la Commune se situe davantage dans la forme que dans le fond. La base des revendications des communards était, en effet, depuis longtemps dans sa réflexion et conforme à ses propositions. Mais l'aspect insurrectionnel du mouvement, s'imposant en dehors du suffrage universel, et la violence qu'il engendrait, cela elle ne pouvait l'admettre. Elle affirme cette conviction le 21 octobre 1871 : 
«Je hais le sang répandu et je ne veux plus de cette thèse : faisons le mal pour amener le bien; tuons pour créer»

Elle ne peut se défaire de son cher rêve de "peuple uni». C'est pour cela qu'elle fit partie des opposants à la Commune, mais pas des plus virulents.

Pourtant elle n'abandonne pas son espoir politique d'une République porteuse d'un monde meilleur socialement. Elle affirme :

«Sachez donc, vous autres, que les républicains avancés sont dans la proportion de un pour cent, sur la surface du pays entier, et que vous ne sauverez la République qu'en montrant beaucoup de patience et en tâchant de ramener les excessifs.»

Quelques mois avant sa mort elle écrit à Flaubert :

«Elle (la République) sera très bourgeoise et peu idéale, mais il faut bien commencer par le commencement. Nous autres artistes, nous n'avons point de patience.»

Plus tard encore, comme en forme de testament, dans une de ses dernières lettres à «son vieux troubadour» elle dit encore :

"Je ne dis pas que l'humanité soit en route pour les sommets, je le crois malgré tout. Mais quant à moi je veux graviter jusqu'à mon dernier souffle, non avec l'exigence de trouver ailleurs une bonne place, mais parce que ma seule jouissance est de me maintenir avec les miens dans le chemin qui monte.»

Et ce "chemin qui monte» George Sand l'a ouvert à ses contemporains avec modestie, sans se départir de ses doutes, et toujours avec courage. Elle fait partie avec d'autres de ces écrivains qui furent au $\mathrm{XIX}^{\mathrm{e}}$ siècle, en quelque sorte, des phares pour éclairer les consciences.

Où sont-ils aujourd'hui? Qui, parmi les artistes de notre temps redonne à l'art ce rôle indispensable de questionner, de remettre en cause, de dénoncer et de proposer pour aller vers un monde meilleur? Qui pour relayer ces créateurs du XIX ${ }^{\mathrm{e}}$ siècle qui ont directement agi sur leur temps? Qui aujourd'hui donne place, dans son œuvre, aux milieux populaires? Pour éluder la question ne dit-on pas tout simplement que la classe ouvrière a disparu? Peut-être pourrions-nous citer Gérard Mordillat pour ses romans ou Robert Guédiguian pour ses films. N'est-ce pas Jacques Demy qui disait «le cinéma français est un cinéma de cadres et les cadres sont vides"?

En guise de conclusion provisoire, relisons Roland Barthes qui écrivait au milieu du $\mathrm{XX}^{\mathrm{e}}$ siècle : 
«Notre littérature serait-elle donc toujours condamnée à ce va-et-vient épuisant entre le réalisme politique et l'art pour l'art, entre une morale de l'engagement et un purisme esthétique, entre la compromission et l'asepsie?

Et si l'avenir du XIX ${ }^{\mathrm{e}}$ siècle était de nous aider à retrouver ce souffle émancipateur de l'art dont nous avons tant besoin?

Georges Buisson, comédien, directeur de théâtre, a été administrateur du Palais Jacques-Cour à Bourges et de la Maison de George Sand à Nohant 